\title{
Índice de actitud hacia la investigación y disposi- ción a la titulación por tesis
}

\author{
Martha Lorena Obermeier Pérez ${ }^{4}$ \\ lorena.obermeiergmail.com \\ Rec. 23/09/2017, Apr. 09/02/2018
}

\begin{abstract}
Resumen:
La Licenciatura en inglés en modalidad virtual está enfocada a la profesionalización de maestros de inglés. Además de la titulación por tesis, la institución acepta otras modalidades, que los alumnos han preferido para titularse. Aun así, los índices de titulación del programa son bajos. En esta investigación, se realizó un análisis cuantitativo para determinar el índice de actitud que tienen los alumnos de la Licenciatura en inglés en modalidad virtual hacia la investigación. Se identificó su concepto sobre las condiciones institucionales respecto al desarrollo de investigación y se detectó su disposición para titularse con la modalidad de tesis.
\end{abstract}

Palabras clave: Investigación - actitud hacia la investigación - titulación por tesis.

\begin{abstract}
:
The on-line Bachelor Degree in English Teaching is focused on training english teachers. Besides obtaining the title writing a dissertation, the institution allows other modalities that the students have chosen. Even thought, the number of students with a title is low. In this research, a quantitative study was conducted to stablish the attitude students have towards research. The concept they have of the conditions in the institution to train researchers and their willingness to write a dissertation were identified.
\end{abstract}

Key words: Research - Attitudes towards research - writing a dissertation.

\section{Introducción}

La falta de titulación por tesis es un fenómeno que afecta a las instituciones educativas en general. En las evidencias prácticas, se manifestó que una universidad pública

4 Docente universitaria especializada en la formación de docentes de inglés, formación de investigadores y tecnología educativa. Integrante del cuerpo académico de investigación Lengua, Cultura y Educación. Actualmente, estudiante del doctorado en educación con especialidad en tecnología educativa y educación a distancia en la Nova Southeastern University en Estados Unidos. 
del sur sureste de México considera a la investigación una de sus actividades sustanciales y genera las condiciones propicias para su desarrollo. Sin embargo, la situación real es diferente, mas no exclusiva de esta institución. Los índices de titulación por tesis son bajos y los alumnos optan por otras modalidades para titularse. En la literatura consultada, se encontró que es un problema que afecta a alumnos de licenciatura y de posgrado tanto en México como en otros países. Existe evidencia de varias acciones remediales diseñadas para resolver los síndromes Todo menos Investigación (TMI) y Todo menos Tesis (TMT), la mayoría con resultados positivos. La situación se agrava cuando se trata de la formación de investigadores en un ambiente virtual, como es el caso de la licenciatura en la que se implementó este estudio. Sin embargo, en esta modalidad también ha habido intervenciones con resultados positivos.

El problema del bajo índice de titulación por tesis afecta no sólo la credibilidad de las instituciones, sino la pertinencia de los programas educativos y al egresado. Un alumno que egresa y no se titula puede llegar a tener problemas personales generados por esta situación. Además de que se cierra el acceso a ciertas oportunidades laborales, se afecta también el campo laboral. La universidad es la formadora de profesionistas y envía egresados al campo laboral con grandes carencias de formación. En el caso de la Licenciatura en inglés, está dirigida a docentes que en su vida profesional deberán hacer investigación para obtener mejores condiciones de contratación, promociones y beneficios laborales. La educación es una vía importante para el desarrollo de un país. Por ello, las instituciones educativas tienen la función de promover la construcción de conocimientos críticos, así como el desarrollo intelectual, afectivo y moral (Graciano, 2013). En la sociedad actual, la educación es una herramienta que permite a los países combatir la pobreza, generar progreso y mejorar los niveles de vida. Las universidades se han enfocado desde sus inicios a las funciones sustantivas de docencia, gestión e investigación. Antes de los años 1990, la función más desarrollada era la docencia.

El modelo de gestión de la educación se modificó a partir de 1990 y se transformó en un modelo de evaluación académica. En México, se crearon organismos como Sistema Nacional de Investigadores (SNI) y Programa para el Desarrollo Profesional Docente (PRODEP) para mejorar las condiciones de producción científica del país y complementar el salario de los profesores investigadores (Pérez \& Guzmán, 2015). Este cambio de gestión modificó la importancia que se atribuía a las funciones académicas, dando mayor importancia a la investigación. Aunado a ese cambio de modelo educativo, la docencia se ha visto modificada a partir del surgimiento del enfoque por competencias. Este enfoque ajusta la formación a las necesidades reales del profesorado o del campo laboral y obliga a una reorganización interdisciplinar que permita la adquisición de competencias. En México, se ha hecho el cambio de los planes de estudio de todos los niveles a este enfoque. Sin embargo, no se ha implementado completamente. Para enseñar por competencias requiere de una serie de recursos con los que las instituciones no cuentan. El cambio a este enfoque ha sido lento y no ha sido bien recibido por los docentes de los distintos niveles educativos.

En este estado actual de la educación, la universidad no puede configurarse como un centro de poder, refugio de mediocres o disimulo del desempleo juvenil (Ferrer, 2016). Debe ser un espacio en el que se dan propuestas sensatas, conocimiento científico. México requiere de docentes que realicen investigación y la fomenten en los alumnos desde el nivel de licenciatura hasta el posgrado. Sin embargo, la mayoría de los programas educativos se enfrenta al problema de los bajos índices de titulación. Para elevarlos, se ha permitido que los alumnos se titulen con otras modalidades no relacionadas a la elaboración de una tesis, 
pero aun así los índices siguen siendo bajos. La eficiencia de los programas disminuye porque no logran graduar la misma cantidad de alumnos que terminan la escolaridad.

\section{Marco teórico}

Desde el origen de las Universidades en el siglo XII, su principal actividad fue la docencia, mientras que la investigación fue una actividad que se realizaba fuera de ellas. En un artículo de Córdoba (2016) se mencionan los modelos universitarios y sus características. El modelo napoleónico o francés formaba profesionales para trabajar en la burocracia de la Francia napoleónica. En este modelo, la investigación era responsabilidad de las academias. Las deficiencias en la formación de este tipo de modelo universitario persisten hasta la actualidad, pues sus estructuras no han sido modificadas por el Estado, las IES ni por los docentes. El segundo modelo universitario es el anglosajón, que surge de las universidades británicas para formar individuos para servir las necesidades de las empresas o del estado. En este modelo prevalece la educación liberal y deja de lado los requisitos necesarios para realizar investigación en la vida profesional, pues rechaza la formación especializada de la investigación y la profesionalización.

El tercer modelo universitario es el creado por Guillermo Humboldt, con influencias de Kant, Hegel, Fichte y Schleiermacher. Este modelo tenía el objetivo de despertar la ciencia en los estudiantes y ayudarlos a desarrollar lo intelectual, por lo que se producía conocimiento y se le incluía en la enseñanza. Se motivaba a trabajar la investigación en todos los campos y a que los profesores fueran investigadores, su labor consistía en transmitir conocimientos, no solamente saberes. La primera universidad que adoptó este modelo fue la Universidad de Berlín en 1810. Los maestros debían ser docentes e investigadores que incluyeran a los alumnos en sus investigaciones y utilizaran la conferencia como estrategia de enseñanza. Se fomentaba que el estudiante pensara, dudara y criticara durante las conferencias y tuviera contacto con expertos en ciencias. Los egresados de este modelo universitario tenían la capacidad para realizar investigación científica, contaban con la formación moral, filosófica, histórica y pedagógica (Córdoba, 2016).

Las universidades latinoamericanas se formaron bajo el modelo napoleónico, enfocado en formar profesionales que fortalezcan la identidad y cultura nacionales. Esta es la razón principal por la cual subsisten las dificultades para formar investigadores y el desarrollar trabajos de investigación. En los planes de estudio de pregrado o licenciatura, se incluyeron los componentes: investigativo, formativo social-humanístico y formación profesional, lo cual fortalece la realización de trabajos de tesis y seminarios en este nivel educativo (Saavedra-Cantor, Muñoz-Sánchez, Antolínez-Figueroa, Rubiano-Mesa, \& Puerto-Guerrero, 2015)). Contrariamente, la Universidad de Humboldt en Alemania se convirtió en un referente de formación investigativa de universitarios. Influyó en algunas de las universidades más prestigiosas de Colombia, razón por la cual se han promovido los semilleros de investigación como estrategia de formación de investigadores. La investigación fue desarrollada en el siglo XX y provocó cambios en las prioridades de las universidades (Nieto, Gómez \& Eslava, 2016). Investigar requiere de un procedimiento sistemático, es una actividad compleja y densa. Se ha convertido en parte fundamental de la formación universitaria y requiere de rigurosidad y excelencia. Por ello, requiere de una disposición anímica hacia el tema de estudio y la motivación es fundamental para realizar el proceso.

Un factor para determinar la calidad de la educación, es la actitud que los estudiantes 
tienen respecto a la investigación científica, elemento fundamental de la formación universitaria. En el período 1938-1941, se integró la docencia- investigación en las universidades, tal como proponía el modelo de Humbolt. Se establece la producción investigativa como factor de evaluación y de ascenso salarial. Actualmente, la ciencia, la tecnología y la innovación se generan gracias a la investigación, por lo cual su desarrollo se fomenta en las instituciones universitarias, escenario propicio para desarrollarla. Actualmente, la investigación es un requisito básico para el desarrollo, para la solución de problemas sociales y una actividad institucional (Córdoba, 2016).

Otro de los indicadores que se consideran para determinar la calidad de las universidades es la producción académica de sus docentes. Recientemente, la política educativa internacional tiende a involucrarlos en la investigación y se busca mejorar la calidad por medio de la formación (Reyes \& Hernández, 2014). El modelo de gestión de la educación se modificó a partir de 1990 y se transformó en un modelo de evaluación académica. Se crearon organismos como SNI y el PRODEP para mejorar las condiciones de producción científica del país y complementar el salario de los profesores investigadores (Pérez \& Guzmán, 2015). A partir de esta transformación académica, se ha dado mayor impulso e importancia a la realización de investigación en las universidades. Para consolidar la investigación, se dan condiciones de financiamiento a nivel nacional y políticas institucionales para apoyar a los académicos.

El índice de titulación es un indicador de eficiencia del programa y es uno de los criterios para la acreditación de un programa académico. El índice de titulación por tesis es bajo en la mayoría de las universidades, por lo que se han permitido otras modalidades para elevarlos y la mayoría de las intervenciones se han realizado en programas presenciales. En el caso de la educación virtual, el conocimiento sobre su eficiencia terminal todavía está poco explorado. Algunos factores que aumentan la eficiencia terminal son la actitud del alumno y su adaptación a la modalidad virtual; las actividades de aprendizaje que fomenten la participación activa; la formación de comunidades de aprendizaje y el empleo de tecnología para desarrollar habilidades cognitivas.

Elizabeth Valarino (como se citó en Ambrosini \& Mombrú, 2017) define el síndrome TMT y TMI como los trastornos, impedimentos, actitudes y deficiencia de destrezas para diseñar, planificar y desarrollar trabajos de investigación, así como el enseñar estos procesos. Esta es una problemática multifactorial, cuyos motivos psicológicos se mezclan con los de carácter social, económico e institucional son generadores de estos síndromes. También se señala la desorientación y el aislamiento que demanda la realización de este tipo de trabajo. Se menciona igualmente el desánimo que produce la falta de apoyo o acompañamiento durante el proceso de escritura. El trabajo de grado no debe ser visto como un requisito de titulación, sino como la oportunidad de investigar un fenómeno y vincularlo a la realidad. La investigación ayuda a pensar en los problemas, detectarlos, describirlos y pensar de forma metodológica cómo solucionarlos. Investigar debería ser inherente a la labor docente, pues el perfil de ambas actividades es el mismo, el de un investigador científico (Gómez, 2013). El TMT aparece en los alumnos que no tiene motivación para realizar una investigación; tienen falta de seguridad en sí mismos; creen que no existe un tema interesante o que consideran que su trabajo no tendrá utilidad. En ocasiones, los índices de titulación son bajos por la falta de dirección de los profesores, quienes no tienen suficiente dedicación a la investigación, publicaciones aprobadas o líneas de investigación establecidas. Al carecer de experiencia y líneas de investigación, muchos profesores se encuentran en la incapacidad de desarrollar habilidades de investigación en los alumnos. Las conductas 
que evidencian el TMI son la postergación, las auto-verbalizaciones negativas, los hábitos inadecuados, las faltas en la estructura del tiempo y las tareas, la ansiedad ante las metas no cumplidas y los bloqueos para escribir. El bloqueo es un sistema de defensa que se activa para aliviar la tensión ante una tarea muy larga. Es también una forma de manejar el pesimismo, el miedo a la crítica, al fracaso o el perfeccionismo. Algunos investigadores no tienen la paciencia para desarrollar un reporte, tienen malos hábitos de trabajo, falta de inspiración para escribir, deficiencia en la escritura o aversión a ella. Las causas por las que los estudiantes no se titulan por tesis son varias y han sido estudiadas por diversos autores. En muchas instituciones educativas, se han realizado incluso propuestas de intervención para solucionar tal problemática. Algunas razones se deben a problemas económicos, responsabilidades profesionales, miedo a presentar un examen profesional, falta de asesores, incapacidad de elección de un tema o desconocimiento del procedimiento (Montesinos \& Domínguez, 2013). También se ha encontrado la falta de interés por desarrollar un trabajo de tesis, relacionada con el tiempo que se le debe dedicar, por lo que eligen modalidades que demanden menor cantidad de tiempo.

Con la intención de elevar los índices de titulación, muchas instituciones educativas permiten que los alumnos se titulen con otras modalidades, como memorias de servicio social, exámenes de conocimientos generales, por promedio o con exámenes a nivel nacional. El hecho de permitir estas modalidades aumenta el índice de titulación, pero agrava el rechazo hacia la realización de investigación o tesis. El síndrome TMT se debe principalmente a las deficiencias para producir textos académicos, a la falta de competencias para hacer investigación y al modelo que se utiliza para enseñar investigación en ciertas instituciones. Isla, Seijo y Espinosa (2013) clasifican las habilidades del investigador en tres tipos: las básicas, las transversales y las específicas. Dentro de las básicas están saber leer o escribir, que permiten desarrollar otras competencias más complejas. Las transversales son la comunicación, el trabajo en equipo, la solución de problemas, la perspectiva amplia, el análisis crítico y la interacción social. Las competencias específicas son las que permiten relacionar los instrumentos con el lenguaje técnico de determinada área. A estas habilidades debería agregarse la capacidad para encontrar información pertinente y reciente, la capacidad crítica y la actitud propicia para la investigación.

La formación investigativa desarrolla una actitud positiva hacia la cultura científica. La investigación incrementa la capacidad de problematizar, analizar críticamente, indagar y reflexionar sobre el impacto de la realidad. Con ella también se fomentan valores como la disciplina, honestidad y perseverancia. Es necesario que se formen estudiantes con actitud positiva hacia la investigación desde los programas de licenciatura. La idea común de que la investigación es difícil o tediosa, cambia cuando se explica su necesidad e importancia en la formación profesional o los méritos de titularse por realizar una tesis. Para generar una actitud positiva hacia la investigación, se sugiere incluir a los alumnos en los grupos de investigación para que estén familiarizados con ellos y con el proceso de trabajo en grupo. También se debe promover la participación de estudiantes en congresos y seminarios y facilitarles oportunidades de becas a posgrados. Una acción indispensable es realizar el rediseño de los planes de estudio para desarrollar la formación de investigadores, ya que la falta de titulación afecta la credibilidad y la acreditación de los programas académicos. El proceso investigativo debe generar un cambio de actitud hacia la investigación para que se disfrute de ella. Para ello, es necesario el acompañamiento del docente y las condiciones institucionales propicias. Un tesista exitoso debe tener un acercamiento afectivo con el tutor durante el proceso de elaboración de la tesis. El 
asesor es una influencia determinante, pues el contacto con él, la admiración y el respeto que se le tiene motiva a los investigadores jóvenes a continuar desarrollando actividades de investigación. Además, el interés por la ciencia se conforma de procesos cognitivos y emocionales, resultado de cursar estudios en instituciones con tradición científica y académica notables. La relación tutor-alumno es importante y deben tener un vínculo académico y emocional (Spinzl, Sosa, González, \& Aquino, 2015). El tutor puede ayudar a cambiar la percepción de que la tesis es una tarea muy grande, si demuestra apertura, facilita información y aporta ideas al trabajo. En el proceso de elaboración de una tesis, los tutores sienten la incertidumbre sobre si su tutoría es adecuada, mientras que los alumnos tienen la incertidumbre de diseñar su investigación y tomar decisiones al respecto (Glasserman \& Ramírez, 2015). Los estudiantes que tienen mayor posibilidad de terminar son aquellos que han tenido experiencia en la investigación y que llegan con una pregunta de investigación. Los criterios de acreditación de los programas educativos exigen la realización de tesis, exigen realizar una actividad compleja porque se espera una transformación conceptual investigativa.

Si bien Elizabeth Valarino inició las investigaciones referentes a las causas que provocan los síndromes TMI y TMT, en el presente trabajo se relacionarán estos conceptos con la teoría del aprendizaje social de Bandura. Esta teoría tiene cierta influencia del conductismo radical de Skinner y agrega el concepto de modelaje (Grusec, 1992), destacando su importancia en la construcción de la auto eficacia. Señala la importancia de los mecanismos cognitivos en el cambio de comportamiento como evidencia de aprendizaje. No se trata de una teoría estática, sino un mecanismo que se desarrolla de manera simple y progresiva. Los cambios en el comportamiento no se dan por los cambios físicos, sino por la interacción y reacción ante los agentes sociales, además de la madurez propia del individuo. El medio actúa en el individuo y éste a su vez en el medio. La personalidad es el resultado de las experiencias de aprendizaje, pero las experiencias están determinadas por la personalidad del individuo.

Los componentes de la teoría socio cognitiva son el modelaje, la activación de procesos internos y el comportamiento independiente, los cuáles deben combinarse con un enfoque pragmático para involucrar a los alumnos en el proceso de investigación, en lugar de que la investigación sea algo que tienen que estudiar para obtener un grado (Kranke, Brown, Atia \& Knotts, 2015). Esta teoría asigna un papel central a los procesos cognitivos, vicarios, auto-regulatorios y auto-reflexivos en el desarrollo y funcionamiento humano. El aprendizaje se da al experimentar de manera directa los efectos de sus acciones y a través del modelaje social. Toda la experiencia que se da con el comportamiento, cognición y aprendizaje afectivo puede aprenderse de manera vicaria, con solo observar a los demás y las repercusiones de sus acciones (Bandura, 2000).

El modelo de Bandura señala que los tres componentes comportamiento, personalidad y factores ambientales se influyen mutuamente. El comportamiento depende de los factores personales y ambientales, pero a su vez los tres son interdependientes (Staddon, 1984). El comportamiento, los factores personales y el medio influyen entre sí, a esto Bandura lo llamó determinismo recíproco (Phillips \& Orton,1983). La persona no reacciona mecánicamente a las influencias del medio, sino que las procesa e influye en ellas. El medio ambiente sirve para desarrollar las funciones de la auto regulación y establecer estándares personales con los cuáles medir su comportamiento. Las habilidades de pensamiento superior se desarrollan en ambientes en los que el aprendizaje es evaluado, implica un reto y se cuenta con las condiciones que lo propicien. El aprendizaje requiere 
de un estímulo externo y de información para almacenar en la memoria, que pueda recuperarse y aplicarse a situaciones cotidianas (Deaton, 2015).

Bandura también sugiere que el aprendizaje por observación tiene un efecto en el observador y éste aumenta si se considera que quien modela el comportamiento tiene una similitud consigo mismo (Deeming \& Johnson, 2009). Ofrece varias evidencias del papel central del aprendizaje por observación en diversas áreas, particularmente en la auto regulación. Los eventos del medio en forma de modelaje, instrucción y persuasión social afectan a la persona, quien a su vez provoca reacciones del entorno dependiendo de su personalidad y características físicas. El comportamiento determina aspectos del entorno al cual el individuo está expuesto y, a su vez, el comportamiento es modificado por el entorno. El concepto de imitación es uno de los temas centrales del enfoque de Bandura, pues el aprendizaje por observación o imitación es una técnica efectiva para la modificación del comportamiento. El individuo tiene procesos cognitivos mediante los cuales las experiencias sociales influyen en su comportamiento y desarrollo. Abstraen e incorporan información de diversas experiencias sociales, como la exposición ante modelos y discusiones verbales (Grusec, 1992).

Los factores que pueden influir en el auto concepto son el modelaje de habilidades cognitivas realizado por sus compañeros, su comparación con el desempeño social de sus compañeros, las interpretaciones de los maestros y sus comentarios sobre su desempeño (Cowan, 2006). El modelaje tiene un papel importante en la creatividad; al observar a modelos con distintos tipos de pensamiento o comportamiento, el observador puede adoptar esos modelos y mezclarlos con sus características personales (Bandura, 2000). Esta teoría enfatiza la importancia de las respuestas por imitación como uno de los mecanismos del aprendizaje social. Las personas observan, imitan y modelan su comportamiento basándose en los demás (Deaton, 2015). El modelaje por parte de los instructores interesa a los estudiantes a que repitan la actividad. Esto los lleva a solucionar deficiencias con nuevas habilidades, mejorando su habilidad de pensar y analizar problemas de manera científica (Kranke, Brown, Atia \& Knotts, 2015). Para Bandura el modelaje es un proceso cognitivo en el que se reconoce al educador como un modelo con virtudes. Los maestros condicionan a los alumnos de manera que finalmente imitan sus roles, por ello también es una estrategia que construye el auto concepto que se tiene de sí mismo en el contexto profesional (Sanderse, 2013).

Los alumnos se desmotivan si consideran que sus maestros no son competentes o si estos no hacen el modelaje de ciertas actividades (Anderson, 1994). Tener modelos competentes a seguir también aumenta la auto eficacia al ayudar a proporcionar conocimientos y desarrollar habilidades. Estos modelos a seguir también pueden persuadir a los demás sobre las formas de obtener éxito en determinadas tareas y ayudarlos a tener resultados positivos en ciertas situaciones en las que normalmente podrían fallar. Los maestros solamente pueden desarrollar en los alumnos características que ellos mismos poseen. Para Bandura, mucho de lo que se aprende se hace a través de procesos en los cuales se aprenden comportamientos, actitudes, valores y creencias mediante la observación de los demás y las consecuencias de sus acciones (Sanderse, 2013). Bandura señala que la auto eficacia es una mezcla de las creencias que regulan el funcionamiento humano a través de los procesos cognitivos, motivacionales, afectivos y procesos decisivos. Las personas basan su vida en sus creencias de auto eficacia, sus objetivos personales y estándares son la base de sus acciones. Se enfatiza el papel de los procesos cognitivos, la observación y la auto regulación. La percepción del individuo sobre su efectividad para 
realizar una tarea determina la facilidad con que se adaptará a un cambio conductual. Las creencias respecto a la auto eficacia provienen de las experiencias previas en la realización de una tarea, de la observación de lo que quienes le rodean son capaces de realizar, de la manera en que los demás le han ayudado a desarrollar un auto concepto positivo y del auto concepto que tienen de sus habilidades y limitaciones. El auto concepto que la persona tiene de sí determina la elección de actividades y medios que le rodean. Bandura define la auto eficacia como la expectativa que la persona tiene de su eficiencia personal y sugiere que la auto eficacia es el factor clave que determina si una persona se comprometerá a cierto comportamiento o actividad.

Un concepto bajo de auto eficacia genera estrés y disminuye el uso de capacidades al enfocarse en sus deficiencias y en resultados negativos, en lugar de enfocarse en estrategias que le permitan alcanzar los objetivos propuestos (Anderson, 1994). Cuando la persona tiene un auto concepto negativo respecto a una situación o se considera ineficiente en la realización de la tarea, esto le genera preocupación y ansiedad, dos condiciones que lo distraen e impiden su correcto desempeño (Grusec, 1992). Quienes dudan de su auto eficacia, dejan de intentarlo pronto; mientras que aquellos que tienen un mejor auto concepto, siguen intentando hasta tener éxito. Tener un concepto alto de la auto eficacia aumenta el interés en la realización de la tarea. Por el contrario, quienes tienen un concepto bajo se alejan de las tareas que consideran difíciles, se rinden rápidamente ante los obstáculos, tienen un compromiso débil con el cumplimiento de los objetivos y se enfocan en sus deficiencias personales (Anderson, 1994). Las personas con poca auto eficacia evitan las tareas difíciles por considerarlas una amenaza, tienen pocas aspiraciones y poco compromiso con el cumplimiento de metas. Se centran en sus inseguridades y miedos, en lugar de buscar soluciones para alcanzar el éxito. El fracaso provoca que pierdan la fe en sus capacidades, pues lo consideran una prueba de su incapacidad. Para tener éxito en la tarea, las personas deben tener un alto sentido de su eficacia con lo que mantienen la perseverancia para finalizar la tarea. Por ello, la auto eficacia alta es necesaria para el buen funcionamiento independientemente de si la tarea se realiza de forma individual o en grupo (Bandura, 2000).

Las personas con mayor confianza en su eficacia suelen establecer retos altos y visualizan escenarios con resultados positivos. Estas personas tienen más recursos cognitivos, flexibilidad estratégica y eficiencia en la administración del medio que los rodea. Consideran que los obstáculos pueden vencerse y planean formas para vencerlos, los consideran un reto. Por lo general, tienen interés en lo que hacen, establecen objetivos altos y tienen compromisos fuertes con la tarea. Se concentran en la realización de la tarea y no en ellos mismos. La mayor parte de la motivación del ser humano se genera con el logro de metas que a la vez generan una mayor motivación (Bandura, 2000). Aunque muchos estudiantes tienen temor a los cursos de investigación, reconocen su importancia para su vida profesional. Entender la importancia de la investigación reduce la ansiedad y aumenta el interés por ella. La teoría de Bandura propone que los individuos aprenden a través de la experiencia directa o al observar el comportamiento en sí. Los enfoques instruccionales que incrementen la auto eficacia de los alumnos impactarán positivamente en su interés por desarrollar investigación (Kranke, Brown, Atia \& Knotts, 2015).

Rojas, Méndez y Rodríguez (2012) realizaron un estudio sobre el Índice de Actitud hacia la Investigación (IAI) de los estudiantes. Estos autores señalan que la actitud no es innata en el sujeto, depende en gran medida de las condiciones mismas del proceso de educación actual del individuo, así como de su trayectoria escolar. La actitud hacia la in- 
vestigación del estudiante es influenciada por los modelos y estructuras investigativos de los centros de formación. Las tres dimensiones que establecen la predisposición hacia la investigación son: el contexto institucional, la calidad de la formación y las motivaciones intrínsecas del estudiante, esta última como clave en el proceso investigativo. El estudio de la actitud hacia la investigación se entiende como la mejor predisposición del estudiante como condición para un mejor proceso de aprendizaje y una mayor probabilidad de formar investigadores en la universidad. En el instrumento diseñado a partir de este constructo, se contemplan tres sub variables que miden el IAI: auto concepto, incidencia de los profesores e incidencia institucional.

\section{Metodología}

El problema de investigación del estudio fue que los egresados de una Licenciatura en inglés, en modalidad virtual no se titulan por tesis, aunque la investigación es una función sustantiva de la universidad que ofrece este programa. Se midió el Índice de Actitud hacia la Investigación (IAI) para determinar si existe correlación con la disposición a la titulación por tesis. El propósito del estudio fue identificar el índice de actitud de los alumnos hacia la investigación, determinar si existía correlación con la disposición para titularse por tesis y conocer el concepto que tienen sobre las condiciones institucionales.

Las preguntas de investigación fueron: ¿Qué índice de actitud tienen los alumnos de la Licenciatura en inglés en modalidad virtual hacia la investigación?, ¿Qué correlación existe entre el índice de actitud hacia la investigación y la disposición para titularse por tesis? y ¿Qué concepto tienen los alumnos de la Licenciatura en inglés en modalidad virtual sobre las condiciones institucionales respecto al desarrollo de investigación y realización de tesis? La población del estudio estuvo conformada por los alumnos de la Licenciatura en inglés en modalidad virtual. La muestra fue dirigida ya que el procedimiento de selección estuvo orientado por las características de la investigación (Hernández Sampieri, Fernández Collado \& Baptista Lucio, 2014) y fue compuesta por los estudiantes del tercero al sexto semestre de la licenciatura. Tuvo un tamaño de 27 alumnos, que se seleccionaron porque las unidades académicas del eje de investigación se incluyen en tercero, quinto y sexto semestre del programa de estudios. Por esta razón, se intuye que ya cuentan con una actitud hacia la investigación y ya han tomado una postura respecto a la modalidad de titulación por tesis. De la muestra seleccionada, se alcanzó el $70.3 \%$ de respuesta. Se aplicó un instrumento para medir el Índice de Actitud hacia la Investigación científica o académica que tienen los alumnos de los semestres tercero a sexto de la Licenciatura en inglés en modalidad virtual. En este caso, el IAI se conforma de su auto evaluación, de la intervención de los docentes y de las condiciones institucionales. El instrumento permitió también obtener información general de los alumnos e información sobre las variables que lo conforman. Posteriormente, con esos datos se pudo determinar si existe correlación entre las variables del estudio. El instrumento está validado, pues fue utilizado previamente en un estudio realizado en Colombia por Rojas, Méndez y Rodríguez (2012). Este estudio se condujo bajo la premisa de que la actitud es fundamental en el acto educativo y que se pueden establecer tendencias midiéndola con escalas correlacionales. El instrumento cuantitativo incluye preguntas con la escala de Likert para medir escalas de actitud de las variables autoevaluación, incidencia de los profesores e incidencia de las condiciones institucionales. Los datos obtenidos del IAI se presentan en la tabla 1, junto con la frecuencia con que se obtuvo cada valor y el porcentaje que representa. En la tabla 2 se presentan los rangos 
de percentiles para determinar si los IAI se ubican en el rango bajo, medio o alto. Como puede observarse en esta última tabla, el 50\% del índice se encuentra en el puntaje de 61.8 del IAI, por lo que la mayoría de los alumnos tienen un índice bajo o medio.

\section{Datos Descriptivos del IAI}

\begin{tabular}{cc}
\hline N estadístico & 19 \\
\hline Rango estadístico & 58.8 \\
Mínimo estadístico & 39.2 \\
Máximo estadístico & 98.0 \\
Media estadística & 64.142 \\
Error estándar & 3.6432 \\
Desviación estándar & 15.8802 \\
Varianza & 252.180 \\
\hline
\end{tabular}

\section{Tabla de Frecuencias del IAI}

Tabla 1

\begin{tabular}{|l|l|c|c|c|c|}
\hline \multicolumn{2}{|c|}{} & Frecuencia & Porcentaje & Porcentaje válido & Porcentaje acumulado \\
\hline Válido & 39.2 & 1 & 5.3 & 5.3 & 5.3 \\
\cline { 2 - 6 } & 46.1 & 1 & 5.3 & 5.3 & 10.5 \\
\cline { 2 - 6 } & 49.0 & 1 & 5.3 & 5.3 & 15.8 \\
\cline { 2 - 6 } & 52.0 & 2 & 10.5 & 10.5 & 26.3 \\
\cline { 2 - 6 } & 53.9 & 1 & 5.3 & 5.3 & 31.6 \\
\cline { 2 - 6 } & 55.9 & 1 & 5.3 & 5.3 & 36.8 \\
\cline { 2 - 6 } & 56.9 & 2 & 10.5 & 10.5 & 47.4 \\
\cline { 2 - 6 } & 61.8 & 2 & 10.5 & 10.5 & 57.9 \\
\cline { 2 - 6 } & 67.6 & 2 & 10.5 & 10.5 & 68.4 \\
\cline { 2 - 6 } & 69.6 & 1 & 5.3 & 5.3 & 73.7 \\
\cline { 2 - 6 } & 70.6 & 1 & 5.3 & 5.3 & 78.9 \\
\cline { 2 - 6 } & 83.3 & 1 & 5.3 & 5.3 & 84.2 \\
\cline { 2 - 6 } & 86.3 & 1 & 5.3 & 5.3 & 94.5 \\
\cline { 2 - 6 } & 90.2 & 1 & 5.3 & 5.3 & 100.0 \\
\cline { 2 - 6 } & 98.0 & 1 & 5.3 & 5.3 & \\
\cline { 2 - 6 } & Total & 19 & 100.0 & 100.0 & \\
\hline
\end{tabular}




\section{Tabla con Rangos de Percentiles del IAI}

\begin{tabular}{ccc}
\hline & Rangos & Total \\
\hline Bajo & $39.2-55.9$ & 7 \\
Medio & $56-67.9$ & 6 \\
Alto & $67.7-98$ & 6 \\
\hline
\end{tabular}

Tabla 3

Para responder la segunda pregunta de investigación, ¿qué correlación existe entre el índice de actitud hacia la investigación y la disposición para titularse por tesis? Se realizó una prueba de chi cuadrado, el resultado fue que estadísticamente no hay correlación porque el valor de chi cuadrada calculada (26.634) es menor al valor de chi cuadrada de tabla (41.33). Los resultados obtenidos se presentan en la tabla 4:

\section{Pruebas de chi-cuadrado}

\begin{tabular}{|l|l|l|c|}
\hline & Valor & df & Significación asintótica (bilateral) \\
\hline Chi-cuadrado de Pearson & $26.634^{\mathrm{a}}$ & 28 & .538 \\
\cline { 2 - 5 } $\begin{array}{l}\text { Razón de verosimilitud } \\
\text { Asociación lineal por lineal }\end{array}$ & 29.194 & 28 & .403 \\
\cline { 2 - 5 } N de casos válidos & 4.303 & 1 & .038 \\
\cline { 2 - 4 } & 19 & & \\
\cline { 2 - 4 } & &
\end{tabular}

Tabla 4

La tercera pregunta fue ¿qué concepto tienen los alumnos de la Licenciatura en inglés en modalidad virtual sobre las condiciones institucionales respecto al desarrollo de investigación y realización de tesis? En la tabla 5, se dan los datos descriptivos del concepto que los alumnos tienen sobre la incidencia institucional. En la tabla 6, se dan las frecuencias de la incidencia institucional y en la tabla 7 los rangos para determinar el índice. En la última tabla, se puede observar que el 50\% del índice se encuentra en el valor 61.1, por lo que los alumnos tienen una actitud baja o media respecto a la incidencia institucional.

\section{Datos Descriptivos de la Incidencia Institucional}

\begin{tabular}{ll}
\hline N estadístico & 19 \\
\hline Rango estadístico & 66.7 \\
Mínimo estadístico & 33.3 \\
Máximo estadístico & 100 \\
Media estadística & 60.92 \\
Error estándar & 4.23 \\
Desviación estándar & 18.47 \\
Varianza & 341.18 \\
\hline
\end{tabular}


Tabla de Frecuencias de la Incidencia Institucional

\begin{tabular}{|c|c|c|c|c|c|}
\hline \multicolumn{2}{|c|}{} & Frecuencia & Porcentaje & $\begin{array}{c}\text { Porcentaje } \\
\text { válido }\end{array}$ & $\begin{array}{c}\text { Porcentaje acu- } \\
\text { mulado }\end{array}$ \\
\hline \multirow{3}{*}{ Válido } & 33.3 & 1 & 5.3 & 5.3 & 5.3 \\
\cline { 2 - 6 } & 38.8 & 2 & 10.5 & 10.5 & 15.8 \\
\cline { 2 - 6 } & 50.0 & 4 & 21.1 & 21.1 & 36.8 \\
\hline & 52.7 & 1 & 5.3 & 5.3 & 42.1 \\
\hline 55.5 & 1 & 5.3 & 5.3 & 47.4 \\
\hline & 61.1 & 1 & 5.3 & 5.3 & 52.6 \\
\hline & 63.8 & 4 & 21.1 & 21.1 & 73.7 \\
\hline 66.6 & 1 & 5.3 & 5.3 & 78.9 \\
\hline & 69.4 & 1 & 5.3 & 5.3 & 84.2 \\
\hline & 86.1 & 1 & 5.3 & 5.3 & 89.5 \\
\hline 100.0 & 2 & 10.5 & 10.5 & 100.0 \\
\hline & Total & 19 & 100.0 & 100.0 & \\
\hline
\end{tabular}

Tabla 6

Tabla con Rangos de Percentiles de la Incidencia Institucional

\begin{tabular}{ccc}
\hline & Rangos & Total \\
\hline Bajo & $33.3-50$ & 7 \\
Medio & $50.1-63.8$ & 7 \\
Alto & $63.9-100$ & 5 \\
\hline
\end{tabular}

Tabla 7

\section{Auto Evaluación -IAE}

Las primeras seis preguntas en escala Likert se refieren al auto concepto que los alumnos tienen sobre sus capacidades respecto a la investigación. La primera es respecto a la satisfacción con el nivel de la carrera, a lo cual los mayores porcentajes se centran en frecuentemente y muy frecuentemente. En la tabla 8 se presentan los datos descriptivos del concepto que los alumnos tienen sobre su auto evaluación respecto a la capacidad de realizar investigación. En la tabla 9, se presentan las frecuencias de la auto evaluación y en la tabla 10 los rangos de percentiles para determinar si el índice es bajo, medio o alto. En esta última tabla, se puede observar que el $50 \%$ del índice se encuentra en el valor $58.3 \%$, por lo que los alumnos tienen mayormente una auto evaluación baja o media respecto a sus capacidades para realizar trabajos de investigación. 


\section{Datos Descriptivos de la Autoevaluación}

\begin{tabular}{ll}
\hline N estadístico & 19 \\
\hline Rango estadístico & 55.6 \\
Mínimo estadístico & 38.8 \\
Máximo estadístico & 94.4 \\
Media estadística & 63.8 \\
Error estándar & 3.21 \\
Desviación estándar & 14.02 \\
Varianza & 196.72 \\
\hline
\end{tabular}

Tabla 8

Tabla de Frecuencias de la Autoevaluación

\begin{tabular}{|c|c|c|c|c|c|}
\hline \multicolumn{2}{|c|}{} & Frecuencia & Porcentaje & $\begin{array}{c}\text { Porcentaje } \\
\text { válido }\end{array}$ & $\begin{array}{c}\text { Porcentaje } \\
\text { acumulado }\end{array}$ \\
\hline Válido & 38.8 & 1 & 5.3 & 5.3 & 5.3 \\
\cline { 2 - 6 } & 50.0 & 1 & 5.3 & 5.3 & 10.5 \\
\cline { 2 - 6 } & 52.7 & 3 & 15.8 & 15.8 & 26.3 \\
\cline { 2 - 6 } & 55.5 & 3 & 15.8 & 15.8 & 42.1 \\
\cline { 2 - 6 } & 58.3 & 2 & 10.5 & 10.5 & 52.6 \\
\hline & 61.1 & 1 & 5.3 & 5.3 & 57.9 \\
\hline 66.6 & 1 & 5.3 & 5.3 & 63.2 \\
\hline & 72.2 & 1 & 5.3 & 5.3 & 68.4 \\
\hline & 75.0 & 3 & 15.8 & 15.8 & 84.2 \\
\hline & 77.7 & 1 & 5.3 & 5.3 & 89.5 \\
\cline { 2 - 6 } & 86.1 & 1 & 5.3 & 5.3 & 94.7 \\
\hline & 94.4 & 1 & 5.3 & 5.3 & 100.0 \\
\cline { 2 - 6 } & Total & 19 & 100.0 & 100.0 & \\
\hline
\end{tabular}

Tabla 9

Tabla con Rangos de Percentiles de la Autoevaluación

\begin{tabular}{lll}
\hline & Rangos & Total \\
\hline Bajo & $38.8-55.5$ & 8 \\
Medio & $55.6-72.2$ & 5 \\
Alto & $72.3-94.4$ & 6 \\
\hline
\end{tabular}

Tabla 10 


\section{Incidencia Profesores -IP}

Las siguientes cinco preguntas se refieren al concepto que los alumnos tienen sobre los docentes. Respecto a la primera pregunta el $36.84 \%$ considera que los maestros a veces confían en sus capacidades para realizar investigación. El $57.89 \%$ menciona que los profesores no exponen en las unidades académicas sus propios trabajos de investigación. Esto puede deberse a que las materias ya se encuentran diseñadas por un experto en contenido y son monitoreadas por un asesor, que no tiene presencia en plataforma más que para resolver dudas o evaluar trabajos. La otra razón puede ser que los maestros no exponen sus investigaciones por carecer de productividad académica o de formación como investigadores.

Existe una discrepancia sobre si los docentes están bien preparados en cuanto a metodología de investigación. Un $26.32 \%$ opina que frecuentemente y otro $26.32 \%$ opina que siempre. Sin embargo, las respuestas de a veces y nunca son altas. De esto se puede sumar los porcentajes de nunca, a veces y frecuentemente y se obtiene que un $63.16 \%$ da una consideración baja a la formación metodológica de los maestros. Solamente un 36.84\% los considera capacitados.

En la última pregunta sobre si los profesores los asesoran bien para realizar investigaciones, el $57.89 \%$ considera que nunca o a veces. Por ello, sería recomendable revisar el apoyo que ofrecen los asesores y su formación tanto en metodología como en manejo de sistemas virtuales. En la tabla 11 se presentan los datos descriptivos del concepto que los alumnos tienen sobre la Incidencia de los Profesores. En la tabla 12, se presentan las frecuencias y en la tabla 13 los rangos de percentiles para determinar si el índice es bajo, medio o alto. En esta última tabla, se puede observar que el 50\% del índice se encuentra en el valor $63.3 \%$, por lo que los alumnos tienen mayormente un concepto bajo o medio respecto a la Incidencia de los Profesores.

\section{Datos Descriptivos de la Incidencia de los Profesores}

\begin{tabular}{cc}
\hline N estadístico & 19 \\
\hline Rango estadístico & 60 \\
Mínimo estadístico & 40 \\
Máximo estadístico & 100 \\
Media estadística & 58.21 \\
Error estándar & 4.52 \\
Desviación estándar & 19.74 \\
Varianza & 389.86 \\
\hline
\end{tabular}

Tabla 11 
Tabla de Frecuencias de la Incidencia de los Profesores

\begin{tabular}{|l|c|c|c|c|c|}
\hline \multicolumn{2}{|l|}{ Válido } & Frecuencia & Porcentaje & $\begin{array}{c}\text { Porcentaje } \\
\text { válido }\end{array}$ & $\begin{array}{c}\text { Porcentaje } \\
\text { acumulado }\end{array}$ \\
\hline \multirow{6}{*}{} & 40.0 & 1 & 5.3 & 5.3 & 5.3 \\
\cline { 2 - 6 } & 43.3 & 1 & 5.3 & 5.3 & 10.5 \\
\cline { 2 - 6 } & 46.6 & 2 & 10.5 & 10.5 & 21.1 \\
\cline { 2 - 6 } & 53.3 & 1 & 5.3 & 5.3 & 26.3 \\
\cline { 2 - 6 } & 56.6 & 1 & 5.3 & 5.3 & 31.6 \\
\cline { 2 - 6 } & 60.0 & 1 & 5.3 & 5.3 & 36.8 \\
\cline { 2 - 6 } & 63.3 & 3 & 15.8 & 15.8 & 52.6 \\
\cline { 2 - 6 } & 66.6 & 2 & 10.5 & 10.5 & 63.2 \\
\cline { 2 - 6 } & 70.0 & 1 & 5.3 & 5.3 & 68.4 \\
\cline { 2 - 6 } & 73.3 & 1 & 5.3 & 5.3 & 73.7 \\
\cline { 2 - 6 } & 90.0 & 1 & 5.3 & 5.3 & 78.9 \\
\cline { 2 - 6 } & 93.3 & 1 & 5.3 & 5.3 & 84.2 \\
\cline { 2 - 6 } & 100.0 & 3 & 15.8 & 15.8 & 100.0 \\
\cline { 2 - 6 } & Total & 19 & 100.0 & 100.0 & \\
\hline
\end{tabular}

Tabla 12

Tabla con Rangos de Percentil de la Incidencia de los Profesores

\begin{tabular}{lll}
\hline & Rangos & Total \\
\hline Bajo & $40-60$ & 7 \\
Medio & $60.1-70$ & 6 \\
Alto & $70.1-100$ & 6 \\
\hline
\end{tabular}

Tabla 13

\section{Incidencia Institucional -IINT}

En la tercera y última parte del instrumento se cuestiona sobre la incidencia institucional y la opinión que los alumnos tienen sobre las condiciones de la institución. $68.42 \%$ considera que no se incentiva la investigación lo suficiente, principalmente si se contrasta con la respuesta dada por los alumnos de que la consideran una actividad importante en su desarrollo profesional. El $73.69 \%$ respondieron que nunca o a veces, se tienen incentivos para que realicen investigación científica. De ello se entiende que los proyectos de investigación no contemplan su inclusión, que no se organizan eventos en los que se les involucre y, en caso de que sí se organicen, la información no llega a los alumnos de la licenciatura virtual.

Respecto a la pregunta sobre si la universidad se preocupa por actualizar el conocimiento científico, los alumnos consideran mayormente que a veces o frecuentemente. Estas dos repuestas fueron las más frecuentes, sumando un $57.9 \%$. Respecto a si los 
alumnos consideran que la universidad les ofrece la infraestructura adecuada para realizar investigación, la mayoría respondió frecuentemente, a veces o nunca. Si se suman estas tres respuestas, se tiene un $84.21 \%$ de alumnos que no consideran que se les ofrezca la infraestructura adecuada. Un 57.9\% considera desconocer el proceso de investigación científica. Finalmente, respecto a si se realizan convocatorias para vincularlos en proyectos de investigación, respondieron que a veces o nunca. Estas dos respuestas más frecuentes suman un $63.16 \%$, mientras que solo el $36.84 \%$ responde que sí se realizan convocatorias regulares.

\section{Conclusiones}

De acuerdo con la teoría de Bandura, el medio tiene influencia en el desarrollo del auto concepto. El medio influye a la vez que es influido por las acciones de las personas. Derivado de esta idea, se deduce que las condiciones institucionales no fomentan el desarrollo de investigación en la institución educativa. Otra de las deducciones preliminares es que los docentes no están ejerciendo el modelaje de acciones relacionadas con la investigación, pues carecen de estas habilidades. La ausencia de este tipo de actividades y condiciones del medio para desarrollarlas sugiere que tanto docentes como alumnos tienen una auto eficacia baja respecto al desarrollo de estas actividades.

Después de realizar una revisión preliminar de la literatura, se intuye que la influencia del medio y el modelaje de los docentes no han favorecido el desarrollo de una auto eficacia alta. Sin embargo, será necesario aplicar los instrumentos necesarios para asignar un valor numérico al concepto de auto eficacia del alumno, a la incidencia de los profesores y a la incidencia institucional. Los resultados de la revisión literaria y el análisis de la información obtenida permitirán sugerir acciones remediales para elevar la auto eficacia, mejorar el modelaje y modificar el contexto institucional.

El presente estudio surgió a partir de la interrogante sobre las razones por las cuales los alumnos egresados de una Licenciatura en modalidad virtual no eligen la titulación por tesis. Para titularse, los estudiantes han venido utilizando otras opciones vigentes en la institución educativa. Sin embargo, el índice de titulación no ha mejorado en las últimas generaciones. Además, para la institución educativa es preocupante que los alumnos evadan esta opción de titulación. En este estudio se presenta información desde la perspectiva de los alumnos sobre cómo funciona el programa educativo. Las razones por las que los alumnos no hacen tesis se deben a la formación que reciben, a la dirección de tesis y al programa (Ochoa \& Cueva, 2017).

Romero-Ortega y Sanz-Cabrera (2017) realizaron una intervención en la cual pudieron vincular el problema de la baja eficiencia terminal con los procesos administrativos y con la formación que reciben los estudiantes. Los alumnos consideran las otras modalidades de titulación como más fáciles, rápidas y menos estresantes que la realización de tesis. Sin embargo, se privan de una experiencia que enriquece su formación. Las habilidades de investigación no se obtienen solamente al realizar una tesis, se obtienen durante todo el proceso de formación universitaria, tanto dentro como fuera de las aulas. Estas modalidades aceptadas duplican las tasas de titulación, pero reducen la cantidad de proyectos de investigación. Es necesario encontrar una forma de mejorar los índices de titulación sin disminuir la cantidad de proyectos que se realizan. Una posible solución sería el incorporar la formación investigativa en las aulas y en las actividades extra curri- 
culares. Los estudiantes deben vincularse en los proyectos que desarrollan los docentes, pues a investigar se aprende investigando (Spinzl, et al., 2015).

Se encontró que un porcentaje elevado de los alumnos no conoce los grupos de investigación de la universidad y otro porcentaje más elevado respondió que no participa en los eventos académicos. De esto se concluye que los grupos de investigación no incluyen alumnos en sus proyectos o que sus productos no son presentados a la comunidad estudiantil. También se puede concluir que la institución educativa no organiza eventos académicos o no están dirigidos a los alumnos. Estos resultados se contraponen con la opinión de los alumnos, pues consideran que la investigación es fundamental para su desarrollo en el campo laboral. Este hallazgo coincide con el encontrado en un estudio realizado por Rico, Garrido y Reveles (2015), ya que también encontraron que un gran porcentaje de los alumnos considera que la investigación es importante para su vida profesional, pero revelan que no existe un buen ambiente institucional para la formación en investigación.

Los alumnos consideran que los profesores solamente a veces confían en sus capacidades para realizar investigación. La mayoría señala que en las unidades académicas no se presentan productos de investigación elaborados por sus docentes. Realizar investigación requiere de una postura auto crítica y la disposición para someter el trabajo al escrutinio de los pares (Elliot, 2015). Esta ausencia de productividad académica en las unidades puede deberse a que no cuentan con ella o a que el modelo educativo del programa no les permite hacer cambios en las unidades académicas, pues fueron previamente diseñadas por un experto en contenido. El trabajo del profesor debe complementarse con las herramientas necesarias, la preparación académica y las condiciones institucionales adecuadas (Moreno, Espinosa, Solano \& Fresán, 2016). Respecto a la importancia de la actividad de los docentes, Castorina (2014) menciona que en algunas universidades se ha generado una fisura, como si enseñanza e investigación estuvieran desligadas. En ocasiones, incluso han llegado a desdeñar al profesor que no investiga, cuando en realidad las universidades necesitan personal tanto con habilidades de docencia como de investigación (López, 2015).

En cuatro de las preguntas de esta sub-variable, la opinión de los alumnos respecto a los maestros es negativa. En una sola pregunta de esta sección, las respuestas fueron altas. Se les inquirió si se les exigían normas metodológicas en la realización de los trabajos y a esto respondieron que siempre. En este caso, puede deducirse que como a los alumnos se les exige el uso del formato APA en sus trabajos, confundieran el uso del formato con la exigencia de normas metodológicas de investigación. Se encontró que no existe una correlación significativa entre este índice y la intención de titularse por tesis. Es importante considerar el hecho de que los procedimientos estadísticos se basan en la variabilidad. En el caso de la información obtenida, puede considerarse que los datos no presentaban variabilidad, por lo que no pudo comprobarse la correlación de las dos variables. Por ello, no se obtuvieron resultados contundentes para probar la hipótesis del estudio. Para poder comprender el origen de la falta de titulación por tesis, sería recomendable incluir otras variables para poder determinar una causa significativa del fenómeno del bajo índice de titulación. Otra de las razones por las que no se pudo establecer la correlación, puede deberse al diseño metodológico utilizado o al tipo de instrumento que se utilizó.

Las respuestas a las seis preguntas sobre condiciones institucionales indican que la mayoría considera que no se incentiva la investigación y que no existen incentivos económicos para los alumnos que realizan investigación. También respondieron que no 
consideran que se cuente con la infraestructura adecuada para la realización de investigación, que no se les enseña el proceso en los cursos regulares y que no hay convocatorias regulares para vincularlos en proyectos. A partir de estas respuestas, se deduce que la institución no cuenta con la infraestructura adecuada para realizar investigación y que no existen los programas de incentivos para los alumnos. Sería importante realizar este mismo cuestionamiento en alumnos de otros programas virtuales, alumnos de la modalidad presencial o de otras áreas de estudio para comparar sus opiniones. Cabe mencionar que los alumnos de la modalidad virtual no tienen un contacto continuo con la institución, por lo que la causa puede ser una comunicación débil entre la coordinación del programa y los alumnos. De ello se deduce que, en caso de que la institución cuente con la infraestructura y los programas de investigación, estos no son compartidos con los alumnos de este programa virtual.

Los resultados encontrados en este estudio contradicen muchas de las sugerencias encontradas en la literatura consultada para formar investigadores. Es evidente que la institución educativa es principalmente de docencia y no de investigación. La actitud de los alumnos hacia la investigación es baja porque no se ha fomentado desde los contenidos de las unidades académicas, desde los profesores ni desde las condiciones institucionales. $\mathrm{Si}$ los profesores tienen productividad, esto no es evidente para los alumnos, pues no presentan sus productos en las unidades académicas. Este hallazgo concuerda con el de un estudio realizado por Rico, Garrido y Reveles (2015), quienes detectaron que tal vez los docentes no tienen oportunidad de mostrar en las materias su formación en investigación o carecen de ella. Partiendo de la opinión de los alumnos, tampoco es claro si la institución organiza eventos o tiene programas de fomento a la investigación. Un bajo porcentaje de los alumnos considera que los docentes no fomentan su participación en eventos académicos, aunque esto puede deberse a una falta de comunicación.

La investigación debe apreciarse como elemento básico para aprender, pues la manera en que se le conciba determinará la actitud hacia ella. Quienes realizan investigación afirman que lo hacen como producto de la formación que tuvieron. Por lo general, los docentes que investigan aseguran sentir mayor importancia, mayor satisfacción y considerarse un modelo a seguir. Por ello, la investigación es el mejor escenario para formar investigadores (Gutiérrez, 2014). Los talleres de metodología deben estar acompañados de prácticas investigativas. Los factores personales como la actitud y la motivación son determinantes para la elaboración de una tesis. Además, los factores institucionales (asesorías, uso de biblioteca, internet, acceso a información) también favorecen la realización de investigación. La cultura de investigación no se improvisa, se genera a partir de cambios administrativos, construcción de políticas que la favorezcan y programas de capacitación profesional en investigación. Modificar una estructura universitaria puede ser complejo debido a los ajustes que deben realizarse en sus áreas, procesos y estatutos. Sin embargo, es necesario realizar cambios graduales para lograr que la universidad sea de investigación y no simplemente de docencia. En el caso de los profesores, es difícil desarrollar la cultura de la investigación por desconocer las metodologías aplicables en sus áreas de conocimiento y carecer de bases de formación. Los talleres de capacitación no son suficientes para darles los conocimientos necesarios como investigadores, pues la mayoría están enfocados en la docencia.

En el caso de la Licenciatura en inglés del estudio, solo se forman universitarios para la docencia, a pesar de que el programa contempla el eje de investigación. Los esquemas de las universidades profesionalizantes ya no tienen cabida en el nuevo modelo uni- 
versitario requerido en la actualidad (Romero-Ortega, \& Sanz-Cabrera, 2017). Estos egresados podrán cumplir con estas funciones, pero difícilmente tendrán oportunidad para desarrollar investigación por falta de capacitación. Esta debilidad no explícita en su formación les aleja de oportunidades (tanto en su calidad de estudiante como de profesores de la enseñanza del inglés) como becas de movilidad, beneficios por productividad académica o promociones para recategorización o mejoras en sus condiciones laborales. Específicamente en México, el 70\% de los empleadores tiene dificultad para encontrar empleados cualificados (Garza-Almanza \& Romero-González, 2016). La situación de la enseñanza de lenguas ha cambiado debido a las exigencias de la sociedad. Cada vez es mayor la presencia de lenguas y culturas en los salones de clase. Esto abre un espacio amplio a la investigación de las exigencias a las que se enfrentarán las nuevas generaciones de profesores. Una posible línea de investigación sería respecto a la elaboración de materiales y planes de estudio de formación específica para futuros profesores de inglés, enfocados en desarrollar pensamiento crítico y reflexivo (González, 2016).

En conclusión, el problema del estudio se relaciona con la teoría de que el modelo napoleónico no forma investigadores (Córdoba, 2016). Se encontró la existencia del síndrome TMI en los maestros y el TMT en los alumnos. Aunque se contempla la investigación en el proyecto académico y en los estatutos, en la práctica no hay evidencias de que se fomenten las actividades relacionadas con la investigación. Se desarrollan principalmente actividades de docencia y no de investigación, pues el modelo educativo del programa de estudios no la fomenta. Para que la universidad mejore niveles de titulación, debe promocionar eventos académicos y dar apoyos a la investigación. Además, es necesario incluir alumnos en los proyectos de servicio social y de los cuerpos académicos. La evaluación de los programas mide hasta qué punto se alcanzan los objetivos planteados en el plan de estudios. Los resultados obtenidos de las evaluaciones permiten tomar decisiones para el cumplimiento de los objetivos y la mejora de resultados. En este estudio se evaluó la actitud hacia la investigación y se encontró la necesidad real de los alumnos de tener este tipo de formación, pues expusieron la importancia de la investigación en su campo laboral. Entre los resultados también se reveló información sobre el proceso de enseñanza-aprendizaje del programa, el cual no contempla la formación en investigación. Los resultados muestran que este proceso debe mejorarse y para ello deben tomarse decisiones respecto a las políticas educativas, al currículum, a la efectividad de las prácticas educativas (Moreno, Espinosa, Solano \& Fresán, 2016).

\section{Bibliografía}

- Ambrosini, C., \& Mombrú, A. (2017). "Bendita tesis: tribulaciones personales e institucionales". Perspectivas Metodológicas, 19(2), 105-118.

— Anderson, M. (1994). Self-efficacy of teacher education students: a study base don Bandura's social cognitive theory (tesis doctoral). Recuperada de ProQuest.

- Bandura, A. (2000). Self-efficacy. In Kadzin A. E. (Ed.), Washington: American Psychological Association, American Psychological Association Oxford University Press. Doi:http://dx.doi.org.ezproxylocal.library.nova.edu/10.1037/10522-094

- Bandura, A. (2000). In Kadzin A. E. (Ed.), Social-cognitive Theory. Washington: American Psychological Association, American Psychological Associa- 
tion Oxford University Press. Doi:http://dx.doi.org.ezproxylocal.library.nova. edu/10.1037/10522-140

- Castorina, J. (2014). Condiciones institucionales y gestión académica de la investigación en la universidad pública. Universidad Jesuita de Guadalajara, 44, 1-14.

- Córdoba, M. (2016). Reflexión sobre la formación investigativa de los estudiantes de pregrado. Revista Virtual Universidad Católica del Norte, 47, 20-37. Recuperado de http://revistavirtual.ucn.edu.co/index.php/RevistaUCN/article/ view/740/12666

- Cowan, T. (2006). A conceptual analysis of Albert Bandura's account of self-efficacy and its educational implications (tesis doctoral). Recuperada de ProQuest.

- Deaton, S. (2015). Social learning theory in the age of social media: implications for educational practitioners. I-manager's Journal of Educational Technology, 12(1), $1-5$.

— Deeming, P., \& Johnson, L. (2009). An application of Bandura’s social learning theory: a new approach to deafblind support groups. Trabajo presentado en JADARA.

- Elliot, J. (2015). Lesson y learning study y la idea del docente como investigador. Revista Interuniversitaria de Formación del Profesorado, 84 (29.3), 29-46.

- Ferrer, M. (2016). Análisis y propuestas de mejora sobre la investigación en las universidades ecuatorianas. Palabra, 16, 180-191.

- Garza-Almanza, V., \& Romero-González, J. (septiembre, 2016). Formación de investigadores emprendedores: prototipo para la universidad mexicana del siglo XXI frente a la era de Donald J. Trump. CULCyT, 13 (60), 4-25.

— Glasserman, L., \& Ramírez, M. (junio, 2015). Formación de Investigadores Educativos mediante el diseño de recursos educativos abiertos y móviles. Revista de Investigación Educativa de la Escuela de Graduados en Educación, 5(10), 36-42. Recuperado de http://rieege.tecvirtual.mx/index.php/rieege/article/view155

— Gómez, X. (2013). Síndromes Universitarios: ¿Por qué el trabajo de grado se torna una pesadilla? Revista de Posgrado FACE-UC, 7(12), 275-287.

- González, J. (2016). Pensamiento y toma de decisiones interactivas del profesor novel de inglés ante los problemas prácticos que encuentra durante el periodo de iniciación profesional. Revista Electrónica Interuniversitaria de Formación del Profesorado, 19(1), 201- 214. DOI: http://dx.doi.org/10.6018/reifop.19.1.194521

- Graciano, M. (2013). La investigación como eje integrador de la formación académica y profesional para el futuro. Revista del Centro de Investigación, 10(40), 69-75.

— Grusec, J. (1992). Social learning theory and the developmental psychology: the legacies of Robert Sears and Albert Bandura. Developmental Psychology, 28(5), 776786.

- Gutiérrez, N. (2014). Producción de conocimiento y formación de investigadores. Sinéctica, 43, 1-16. Recuperado de http://www.sinectica.iteso.mx/articulo/?id=43_produccion_de_conocimiento_y_formacion_de_investigadores

- Hernández Sampieri, R., Fernández Collado, C., \& Baptista Lucio, P. (2014). Metodología de la investigación. México, D.F.: McGraw-Hill Education. 
— Isla, M., Seijo, J., \& Espinosa, F. (julio, 2013). Consideraciones para el desarrollo de capacidades de investigación en universidades privadas. Revista del Centro de Investigación, 10(40), 5-13.

— Kranke, D., Brown, J., Atia, M., \& Knotts, G. (2015). A pedagogical approach to engaging social work students in research. Social Work Education, 34(7), 895-901.

- López, E. (2015). Conectando investigación y docencia en la universidad: teaching research nexus. Teoría Educativa, 27(2), 203-220.

— Montesinos, S., \& Domínguez, J. (2013). Análisis y diseño de un sistema de información como propuesta de metodología para realización de tesis de licenciatura. Revista Internacional de la Educación en Ingeniería, 6(1), 8-13.

— Moreno, T., Espinosa, M., Solano, E., \& Fresán, M. (2016). Evaluación de un modelo educativo universitario: una perspectiva desde los actores. Revista Iberoamericana de Evaluación Educativa, 9(2), 29-48.

— Nieto, D., Gómez, N., \& Eslava, S. (2016). Significado psicológico del concepto investigación en investigadores. Diversitas- Perspectivas en Psicología, 12(1), 109121.

- Ochoa, L., \& Cueva, A. (2017). El bloqueo en el proceso de elaboración de una tesis de maestría: angustias y desazones percibidas por sus protagonistas. Lenguaje, 45(1), 61-87.

- Pérez, A., \& Guzmán, M. (2015). Narrativa de los profesores investigadores en torno a las tensiones generadas por las condiciones laborales y evaluación de la productividad académica. Administración y Organizaciones, 18(34), 93-112.

- Phillips, D., \& Orton, R. (1983). Theoretical notes. The new causal principle of cognitive learning theory: perspectives on Bandura's "reciprocal determinism". Psychological Review, 90(2), 158-165.

— Reyes, M., \& Hernández, E. (marzo, 2014). Productividad y condiciones para la investigación: el caso de los profesores de lenguas extranjeras. Sinéctica, 42, 1-17. Recuperado de http://www.sinectica.iteso.mx/articulo/?id=42_productividad_y_ condiciones_para_la_investigacion_el_caso_de_los_profesores_de_lenguas_extranjeras

— Rico, M., Garrido, N., \& Reveles, A. (2015). Las actitudes hacia la investigación en el posgrado de la FECA-UJED. Revista Global de Negocios, 3(5), 71-84

— Rojas, H., Méndez, R., \& Rodríguez, A. (julio, 2012). Índice de actitud hacia la investigación en estudiantes de nivel de pregrado. Entramado, 8(2), 216-229.

— Romero-Ortega, A., \& Sanz-Cabrera, T. (enero, 2017). ¿Tesis o examen de grado? Un dilema para la formación investigativa. Alteridad, 12(2), 238-247.

- Saavedra-Cantor, C. J., Muñoz-Sánchez, A. I., Antolínez-Figueroa, C., Rubiano-Mesa, Y. L., \& Puerto-Guerrero, A. H. (2015). Semilleros de investigación: Desarrollos y desafíos para la formación en pregrado. Educ, 18(3), 391-407. DOI: 10.5294/edu.2015.18.3.2

- Sanderse, W. (2013). The meaning of role modelling in moral and carácter education. Journal of Moral Education, 42(1), 28-42.

- Spinzl, C., Sosa, D., González, L., \& Aquino, B. (junio, 2015). A investigar se aprende investigando. Programa de jóvenes investigadores. Sinéctica, 44, 1-11. 
Recuperado de http://www.sinectica.iteso.mx/articulo/?id=44_a_investigar_se_ aprende_investigando_programa_de_jovenes_investigadores

— Staddon, J. (1984). Social learning theory and the dynamics of interaction. Psychological Review, 91(4), 502-507. 